\title{
Modelling length-at-age variability under irreversible growth
}

\author{
William S.C. Gurney, Graham Tyldesley, Simon N. Wood, Philip J. Bacon, \\ Michael R. Heath, Alan Youngson, and Anton Ibbotson
}

\begin{abstract}
In this paper, we describe a discrete-time formalism for describing the dynamics of the size-at-age distribution of a cohort of individuals exhibiting irreversible von Bertalanffy growth in a statistically uniform random environment. This formalism yields a highly efficient numerical implementation, which is particularly suited to automatic optimization. In the special case where mortality is sufficiently size-independent not to vary substantially across the bulk of the size distribution at any given age, we can further increase this efficiency by deriving compact update rules for the mean and coefficient of variation of size-at-age. In this case, we also demonstrate that the depensatory effect of random growth variability and the compensatory effect of deterministic von Bertalanffy growth balance to yield an attracting (initial condition independent) trajectory of mean length and length coefficient of variation against age. We demonstrate the applicability and extensibility of this formalism by two exemplary applications — juvenile salmonids and demersal cod.
\end{abstract}

\begin{abstract}
Résumé : Nous présentons dans ce travail un formalisme à temps discret pour décrire la dynamique de la distribution de la taille en fonction de l'âge chez une cohorte d'individus qui ont une croissance de type von Bertalanffy irréversible dans un environnement uniformément aléatoire du point de vue statistique. Ce formalisme permet une application numérique très efficace qui se prête particulièrement bien à une optimisation automatique. Dans le cas particulier où la mortalité est suffisamment indépendante de la taille pour ne pas varier essentiellement sur la plus grande partie de la distribution des tailles à chacun des âges, il est possible d'augmenter l'efficacité en élaborant des règles compactes de mise à jour pour la moyenne et le coefficient de variation de la taille en fonction de l'âge. Dans ce cas, nous démontrons que l'effet d'allee provoqué par la variabilité aléatoire de la croissance et l'effet compensatoire de la croissance déterministe de type von Bertalanffy s'équilibrent pour donner une trajectoire attractive (indépendante des conditions initiales) de la longueur moyenne et du coefficient de variation de la longueur en fonction de l'âge. Nous démontrons que ce formalisme peut être étendu en l'appliquant à deux exemples — les jeunes saumons et les morues démersales.
\end{abstract}

[Traduit par la Rédaction]

\section{Introduction}

An individual's calendar age always increases at the same (constant) rate, so the dynamics of population age structure are extremely simple. In contrast, the somatic growth rate of apparently similar individuals often varies widely (Sebens 1987; Pfister and Peacor 2003), so understanding the dynamics of population size structure is a much greater theoretical challenge.

Population size structure often plays a key role in determining dynamics, influencing average fecundity (e.g., Marteinsdottir and Begg 2002); interspecific interactions, such as predation (e.g., Wootton 1992); and intraspecific interactions such as cannibalism (e.g., DeAngelis et al. 1979; Brunkow and Collins 1998). In exploited populations, size structure changes can have substantial economic as well as ecological impacts (Rose 2004).

Ricker (1958) distinguishes between growth compensation (cohort size-at-age variability decreases with time or age) and growth depensation (cohort size-at-age variability increases with age). Pfister and Stevens (2002) identify three classes of mechanism that can lead to growth depensation: size dependence of growth rate, individual-to-individual variability in growth efficiency, and statistically independent

Received 5 September 2006. Accepted 17 December 2006. Published on the NRC Research Press Web site at http://cjfas.nrc.ca on 2 May 2007.

J19516

W.S.C. Gurney. ${ }^{1}$ Department of Statistics and Modelling Science, University of Strathclyde, Glasgow G1 1XH, Scotland; and Fisheries Research Services (FRS) Marine Laboratory, Aberdeen AB11 9DB, Scotland.

M.R. Heath. Fisheries Research Services (FRS) Marine Laboratory, Aberdeen AB11 9DB, Scotland.

G. Tyldesley. Department of Statistics and Modelling Science, University of Strathclyde, Glasgow G1 1XH, Scotland.

S.N. Wood. Department of Mathematics and Statistics, University of Bath, Bath BA2 7AY, United Kingdom.

P.J. Bacon and A. Youngson. FRS Freshwater Fisheries Laboratory, Faskally, Pitlochry PH16 5LB, Scotland.

A. Ibbotson. Centre for Ecology and Hydrology (CEH), Winfrith Technology Centre, Winfrith Newburgh, Dorchester, Dorset DT2 8ZD, United Kingdom.

${ }^{1}$ Corresponding author (e-mail: bill@stams.strath.ac.uk). 
(uncorrelated) stochastic variation in individual growth performance.

The first two of these classes (deterministic size dependence of growth and fixed individual-to-individual differences) yield relatively benign mathematical representations and are thus the most highly represented in the literature (e.g., DeAngelis and Huston 1987; de Roos et al. 1992; Pfister and Peacor 2003). Stochastic variations in individual growth that are uncorrelated with those experienced by others in the population complicate the analysis considerably. Their obvious importance has led to the widespread use of individual-by-individual simulations to investigate their detailed implications (e.g., Pfister and Stevens 2002; Fujiwara et al. 2004, 2005).

Practical difficulty in fitting individual-by-individual simulations to observational data has led to recent interest in more analytic approaches. Bardos (2005) describes the application of a probabilistic variant of the Gompertz (1825) model to size-increment data for abalone (Haliotus sp.). Gudmundsson (2005) has fitted an heuristically derived stochastic variant of the von Bertalanffy model (von Bertalanffy 1938) to length-at-age statistics for Icelandic Atlantic cod (Gadus morhua).

Gurney and Veitch (2007) have proposed a unified framework, employing the coefficient of variation (CV) as the key measure of length-at-age variability, within which all three sources of size-at-age variability can be represented. The central conclusions of this work are that uncorrelated stochastic resource variabity is always depensatory, but deterministic growth is compensatory unless the growth rate increases faster than exponentially with size. Further, the combination of stochastic depensation with compensatory deterministic dynamics implies that a growing cohort will exhibit an initial-condition-independent trajectory of $\mathrm{CV}$ against length.

Although the continuous size-time framework proposed by Gurney and Veitch (2007) is suited to analytic investigation, it is a rather cumbersome vehicle for simulation and hence for fitting to observational data. Moreover, where growth is irreversible, its key dynamic equation is an approximation - albeit a good one for many realistic cases. In this paper, we propose a discrete size-time formalism, which exactly represents irreversible growth and also results in a very economical numerical scheme, thus making it particularly suited to data fitting. In the special case where mortality is size-independent, we are able to derive explicit update rules for the moments of the size-at-age distribution, thus gaining further increases in computational efficiency as well as considerable insight into the influence of particular parameter choices on model predictions.

To illustrate the utility of this formalism, we fit length-atage data sets: for salmonids growing in two contrasting UK rivers and for Atlantic cod caught by the International Bottom Trawl Surveys (IBTS) in European shelf waters close to the UK. In each case the model is able to reproduce key qualitative and quantitative features of the observed lengthat-age statistics, and we can observe regional and speciesspecific changes in the best-fit parameters that are compatible with known features of the biogeography of the species concerned.

\section{The model}

Here we develop an exact representation of the age-size dynamics of a cohort of individuals, each exhibiting irreversible growth according to the pattern proposed by von Bertalanffy (1938). Our central assumption will be that the cohort consisists of a group of functionally identical individuals who are exposed to a statistically uniform environment. Size-at-age variability is thus a product of the combined effects of variability in size at recruitment and individual-toindividual variability in growth rate. The model we present is related to that described by Gurney and Veitch (2007) but differs in two key aspects: first, its representation of irreversible growth is exact rather than approximate; second, it implies a considerably more economical numerical scheme.

To set the context for this presentation, we first set out an energetic derivation for the deterministic model of individual growth proposed by von Bertalanffy (1938). This derivation is given in more detail by Gurney and Nisbet (1998), who consider reproductively inactive individuals with carbon weight $(W)$, which changes at a rate given by the difference between assimilation and maintenance rates ( $A$ and $M$, respectively), so that

$$
\mathrm{d} W / \mathrm{d} t=A-M
$$

They then make two further assumptions. First, $M$ is proportional to $W$ (with a constant of proportionality $m$ ) so that

$$
M=m W
$$

Second, $A$ scales with the square of body length $L$ (with constant of proportionality $a$ ) and has a Holling type II dependence on food abundance $F$ (with half saturation $F_{\mathrm{H}}$ ) so that

$$
A=a L^{2} \phi(F) \quad \text { where } \quad \phi=F /\left(F+F_{\mathrm{H}}\right)
$$

A final assumption that $W$ is proportional to $L^{3}$ (with a constant of proportionality $w$ ) then implies that eq. 1 can be recast to tell us the rate of change of length in terms of its current value and food abundance. At this point, we incorporate our key assumption that while weight can both increase and decrease, animals whose weight is decreasing retain their current length. Using the notation $[x]^{+}=\max (0, x)$ and defining $\alpha \equiv a /(3 w)$ and $\mu \equiv m / 3$ to make the result as compact as possible, we arrive at the following dependence of individual growth rate on current length and food environment:

$$
\mathrm{d} L / \mathrm{d} t=[\alpha \phi(F)-\mu L]^{+}
$$

Our stochastic extension of this model assumes that individual-to-individual variability in growth rate is the product of stochastic variability in food availability, $F$, both in time and between individuals. As a modelling approximation, we assume that the value of $F$ experienced by each individual remains constant for periods of length $\tau$ (which we call the growth correlation time). We further assume that $F$ takes only values that are either large enough or small enough compared with $F_{\mathrm{H}}$ for $\phi$ to be regarded as either 1 or 0 . Finally, we assume that in each growth period, $\phi$ takes the value 1 with probability $p$ and 0 with probability $1-p$.

This picture of feeding and growth in a random environment necessarily relies on a number of fairly heroic biologi- 
cal assumptions. Two of the more egregious of these are that (i) an individuals feeding rate is assumed to be completely correlated (i.e., constant) within the growth correlation time and not at all correlated thereafter, and (ii) any individual who was unable to feed during one growth period can start growing instantaneously upon entering a growth period in which $\phi=1$. Both these assumptions are clearly wrong in detail and must stand or fall on the ability of the resulting formalism to mimic real observations.

For compactness, as well as consistency with the energy budget literature, we define $L_{\infty}$ to represent the asymptotic length of an individual growing with $\phi=1$, thus

$$
L_{\infty}=\alpha / \mu
$$

Hence, the growth of an individual with length $L_{t}$ at time $t$ is described during the time increment $t \rightarrow t+\tau$ by

$$
\mathrm{d} L / \mathrm{d} t= \begin{cases}\mu\left(L_{\infty}-L\right) & \text { with probability } p \\ 0 & \text { with probability } 1-p\end{cases}
$$

where

$$
\text { (7) } \quad L(t)=L_{t}
$$

We note that provided $p>0$, eq. 6 implies that any individual who survives long enough must eventually reach the food-independent asymptotic length defined by eq. 5. This contrasts markedly with the conventional deterministic picture of von Bertalanffy growth under constant food conditions, where asymptotic length is found to be proportional to food abundance. However, where the assimilation rate varies stochastically within fixed bounds, it is clear that an irreversible growth process must eventually take all individuals to the asymptotic length associated with constant growth at the upper bound of the assimilation rate (i.e., $L_{\infty}$ ).

Having established our picture of the dynamics of individual growth, we now turn our attention to the dynamics of the cohort. Although eq. 4 allows us to distinguish individuals entirely on the basis of their length, the key to a mathematically and computationally tractable representation is to transform $L$ into a new variable:

$$
q=-\ln \left[L_{\infty}-L\right]
$$

which we shall refer to as the development index. Given our assumption that $\phi$ takes the values 1 and 0 , with probabilities $p$ and $1-p$, respectively, we see that during any period $t \rightarrow t+\tau$, the rate of change of the development index is

$$
\mathrm{d} q / \mathrm{d} t= \begin{cases}\mu & \text { with probability } p \\ 0 & \text { with probability } 1-p\end{cases}
$$

We now use $n_{q, t}$ to denote the number of individuals in the cohort with development index in the range $q \rightarrow q+\mu \tau$ at time $t$. Equation 9 shows that any individual with $q$ in $q \rightarrow$ $q+\mu \tau$ at time $t$ that is still alive at time $t+\tau$ will, at that time, have $q$ in $q+\mu \tau \rightarrow q+2 \mu \tau$ (with probability $p$ ) or will have an unchanged development index (with probability $1-p$ ). Hence, if we use $S_{q, t}$ to denote the probability that an individual with $q$ in $q \rightarrow q+\mu \tau$ at time $t$ is still alive at time $t+\tau$, we can see that

$$
n_{q, t+\tau}=(1-p) S_{q, t} n_{q, t}+p S_{q-\mu \tau, t} n_{q-\mu \tau, t}
$$

We see immediately that eq. 10 exactly describes the dynamics of the size distribution during a growth process in which individuals cannot shrink, but must either retain their current size or exhibit positive growth.

Equation 10 provides a very simple route to simulating the trajectory of the development index distribution. However, the development index is neither directly observable nor intuitive, so we normally calculate either a length distribution or summary statistics, such as mean length.

The easiest relationship to derive is that between the development index distribution and simple summary statistics. We can see from eq. 8 that an individual with development index $q$ has length $L_{q}$, where

$$
L_{q}=L_{\infty}-\mathrm{e}^{-q}
$$

The expectation values of $L$ and $L^{2}$ over the population length distribution at time $t$ are thus given by sums over all values of $q$, thus

$$
\hat{L}_{t}=\frac{\sum L_{q} n_{q, t}}{\sum n_{q, t}}, \quad \hat{L}_{t}^{2}=\frac{\sum L_{q}^{2} n_{q, t}}{\sum n_{q, t}}
$$

The variance $\left(\sigma_{t}^{2}\right)$ and $\mathrm{CV}\left(c_{t}\right)$ of $L$ at time $t$ are then calculated from

$$
\sigma_{t}^{2}=\hat{L}_{t}{ }_{t}-\left(\hat{L}_{t}\right)^{2}, \quad c_{t}=\frac{\sqrt{\sigma_{t}^{2}}}{\hat{L}_{t}}
$$

To transform the development index distribution into a length distribution, we note that if $N_{L, t}$ represents the number of individuals with lengths in $L \rightarrow L+\mathrm{d} L$ then

$$
N_{L_{q}, t}=\frac{n_{q, t}}{\mathrm{~d} L / \mathrm{d} q}=\mathrm{e}^{q} n_{q, t}
$$

We illustrate the result of a typical calculation using this formalism (Fig. 1) and in particular the raw development index distribution calculated from repeated applications of eq. 10 (Fig. 1a). The initial, very narrow $q$ distribution is spread steadily by the diffusive effect of variable growth rate. The same results transformed to a length distribution using eq. 14 (Fig. 1b) show the combined effect of the compensatory deterministic dynamics (bringing individual growth trajectories closer) and the depensatory effect of growth rate variability (spreading them apart).

Despite the formal differences between the model described here and that analysed by Gurney and Veitch (2007), their work leads us to expect that when a system shows compensatory deterministic dynamics combined with stochastically varying growth, the outcome will be that the $\mathrm{CV}$ of length will rapidly converge to an initial conditionindependent trajectory (Figs. $1 c$ and $1 d$ show that this does indeed occur).

\section{Size-independent mortality}

\section{Length-at-age statistics}

If the step-to-step survival is size-independent, that is $S_{q, t}=S_{t}$, then multiplying both sides of eq. 10 by $L_{q}$ and summing over all $q$, using eq. 11 to relate $L_{q-\mu \tau}$ to $L_{q}$, shows that 
Fig. 1. Growth of immortal individuals under constant conditions is illustrated. The cohort shows immortal individuals exhibiting von Bertalanffy growth (eq. 4 with a time step of 1 day) with $\alpha=12.5, \mu=0.05$, and $p=0.5$. (a) Development index distributions are shown for the following times ( $t$ in days; peaks from left to right): $t=10$ (dashed), $t=20$ (dot-dashed), $t=40$ (longer-dashed), $t=80$ (dot-dot-dashed), and $t=120$ (solid) for a run with all individuals having initial length 50. (b) Implied length distributions for the same run at the same times. (c) Mean length against age for two runs: one with all individuals of initial length $50 \mathrm{~mm}$ (solid) and the other with initial lengths normally distributed with a mean of $50 \mathrm{~mm}$ (dotted). Note that these two lines are coincident, so only the solid line is visible. $(d)$ Coefficient of variation $(\mathrm{CV})$ for length plotted against age for the same two runs.

(a)

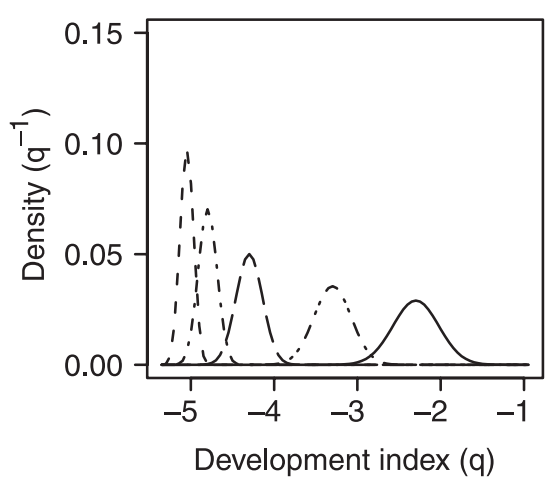

(c)

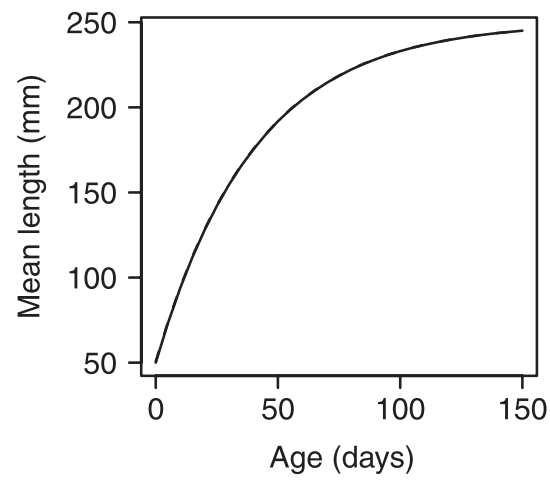

(15)

$$
\hat{L}_{t+\tau}=\hat{L}_{t}+\gamma \tau\left(L_{\infty}-\hat{L}_{t}\right)
$$

where, for compactness, we define a maximum relaxation rate $(\xi)$ and an expected relaxation rate $(\gamma)$ as

$$
\xi=\left(1-\mathrm{e}^{-\mu \tau}\right) / \tau \quad \text { and } \quad \gamma=p \xi
$$

A very similar, although rather more algebraically taxing, argument yields an update rule for $\hat{L}^{2}$, thus

$$
\hat{L}_{t+\tau}^{2}=\hat{L}_{t}^{2}+\gamma \tau\left[\xi \tau L_{\infty}^{2}+2(1-\xi \tau) L_{\infty} \hat{L}_{t}-(2-\xi \tau) \hat{L}_{t}^{2}\right]
$$

In cases where mortality is size-independent, eqs. 15 and 17 provide a noticeably more efficient route to results such as those in Figs. $1 c$ and $1 d$ than a repeated iteration of eq. 10.

We note that the trajectory produced by iterating eqs. 15 and 17 from a starting point $\left\{\hat{L}_{0}, \hat{L}_{0}^{2}\right\}$ with $\{\mu, p, \tau\}=\left\{\mu_{1}, p_{1}\right.$, $\left.\tau_{1}\right\}$ is not uniquely related to the parameters. For example, eq. 15 shows that the mean length trajectory from $\hat{L}_{0}$ with $\{\mu, p, \tau\}=\left\{\mu_{2}, p_{2}, \tau_{1} / 2\right\}$ is identical to that produced with $\{\mu, p, \tau\}=\left\{\mu_{1}, p_{1}, \tau_{1}\right\}$ at the times when the trajectory with $\tau=\tau_{1}$ is defined (that is, at $t=n \tau_{1}$ with $n$ being a positive integer), provided only that the two parameter sets imply values of $\gamma$ (say $\gamma_{1}$ and $\gamma_{2}$ ), which satisfy (b)

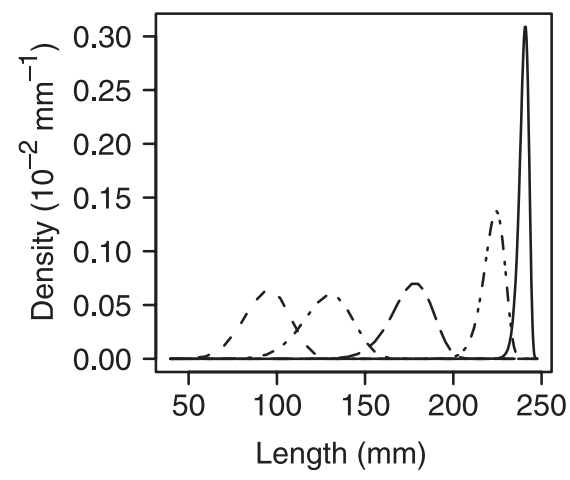

(d)

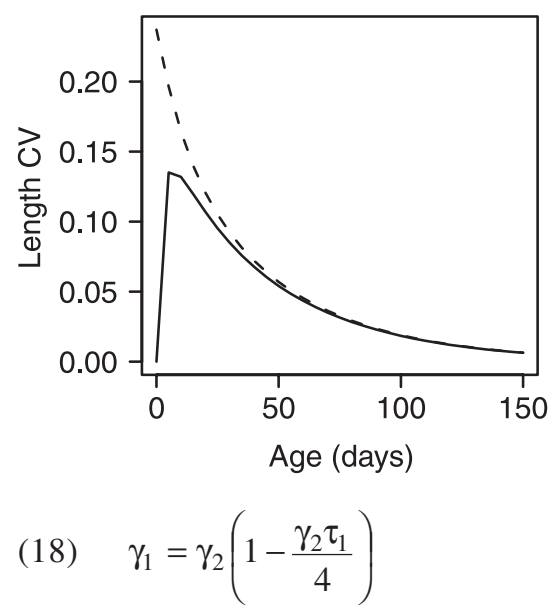

A similar, but more algebraically taxing, argument yields an independent requirement on $\gamma$ and $\xi$ for the process to yield an identical trajectory for $\hat{L}^{2}$. Taken together, these define values $\mu_{2}$ and $p_{2}$ such that $\left\{\mu_{2}, p_{2}, \tau_{1} / 2\right\}$ implies an identical trajectory to $\left\{\mu_{1}, p_{1}, \tau_{1}\right\}$.

A similar argument can be developed for any other value of $\tau$ that is a submultiple of $\tau_{1}$. We conclude that to define a unique parameter set yielding any specified trajectory, we must first select a value for $\tau$ and then find the unique values of $p$ and $\mu$ corresponding to that trajectory. This has obvious implications for the data-fitting process that occupies the latter part of this paper.

\section{Quasi-stationary CV}

Our simulations suggest that in this model, as in its continuous-time relative investigated by Gurney and Veitch (2007), the combination of compensatory deterministic dynamics and random growth rate variation will lead to a quasi-steady-state CV for size-at-age. Recalling the defini- 
tions in eq. 13, we see that the $\mathrm{CV}$ remains constant with age (time) if

$$
\frac{\hat{L}_{t+\tau}-\left(\hat{L}_{t+\tau}\right)^{2}}{\left(\hat{L}_{t+\tau}\right)^{2}}=\frac{\hat{L}_{t}^{2}-\left(\hat{L}_{t}\right)^{2}}{\left(\hat{L}_{t}\right)^{2}}
$$

Substitution from eqs. 15 and 17, followed by a considerable quantity of routine algebra, yields an expression for the quasi-steady-state value of $\hat{L}^{2}$, which after a small amount of further manipulation gives the quasi-steady-state coefficient of length variation as

$$
c_{t}^{*}=\sqrt{\frac{(\xi-\gamma)\left(L_{\infty}-\hat{L}_{t}\right)^{2}}{\gamma\left(L_{\infty}-\hat{L}_{t}\right)^{2}-\xi\left(\hat{L}_{t}\right)^{2}+(2 / \tau) L_{\infty} \hat{L}_{t}}}
$$

We see from eq. 16 that when $\mu \tau<<1, \xi \approx \mu$ and $\gamma \approx \mu p$, so that both are formally indendent of $\tau$. In the more general case, we expect that these two coefficients will depend only weakly on $\tau$. Thus we expect that for any given length, the quasi-stationary $\mathrm{CV}$ increases as the difference between $\xi$ and $\gamma$ increases - that is, as $p$ decreases. We also see that it increases slowly with $\tau$ unless $\mu \tau<<1$, when it becomes proportional to $\sqrt{\tau}$.

Equation 20 also tells us that the quasi-steady-state CV for length-at-age falls with increasing mean length (and hence with age), going asymptotically to 0 as $\hat{L}$ approaches $L_{\infty}$. We thus expect that the trajectory of $\mathrm{CV}$ against age predicted using eqs. 15 and 17 will be considerably above that implied by eq. 20. Comparison of the values predicted by eq. 20 with values from explicit simulations such as those in Fig. 1 confirms this supposition. Typical erors are $10 \%-$ $30 \%$, so we conclude that while eq. 20 plays an important role in allowing us to understand the way in which model parameters control the predicted variability and can provide a useful approximation to likely observed variability, it cannot provide a route to accurate estimates of the length-at-age variability implied by any given set of model parameters.

\section{Salmonids in two UK rivers}

\section{The data}

To illustrate the formalism described above, we examine three sets of observations of wild juvenile salmonids growing in a river environment. The earliest data set concerns Atlantic salmon (Salmo salar) parr observed between 1998 and 2003 by staff from the Fisheries Research Services Freshwater Laboratory during regular electrofishing surveys in the Girnock Burn, an upland tributary of the River Dee in northeast Scotland. A full description of the site (at $57^{\circ} 00^{\prime} \mathrm{N}, 3^{\circ} 08^{\prime} \mathrm{W}$ ) can be found in Buck and Hay (1984). On first capture, individuals longer than $70 \mathrm{~mm}$ were scalesampled and marked before being weighed, measured, and released. Recaptured individuals were identified, weighed, and released. Length/weight trajectories from long-surviving individuals observed in the first year of this programme have been modelled by Jones et al. (2002), and their growth patterns have been subjected to statistical analysis by Bacon et al. (2005). In the present work, we use observations of length-at-age for the 1997 hatch class (Fig. 2c).
Fig. 2. Length (mm) against age (days from 1 January in year of hatch) for (a) brown trout (Salmo trutta) observed during 20022003 in the River Frome, (b) trout from the 2002 hatch observed in the River Girnock, and (c) Atlantic salmon (Salmo salar) parr from the 1997 hatch observed in the River Girnock.

(a)

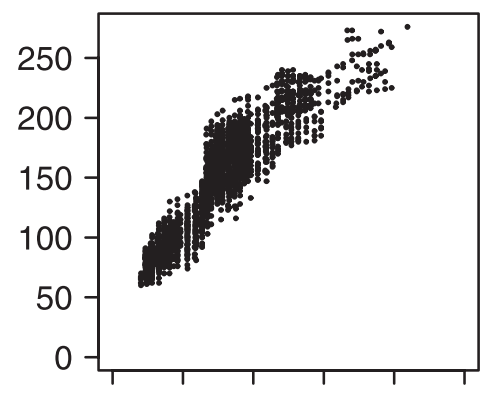

(b)

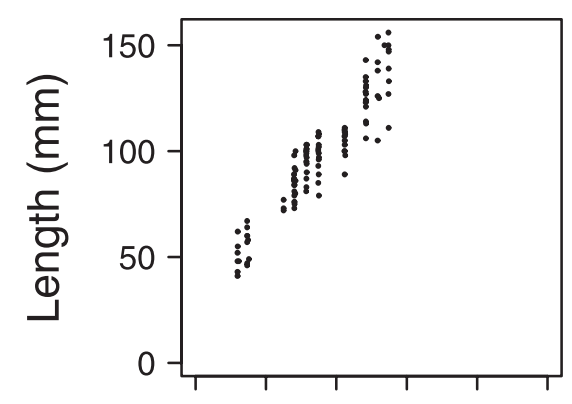

(c)

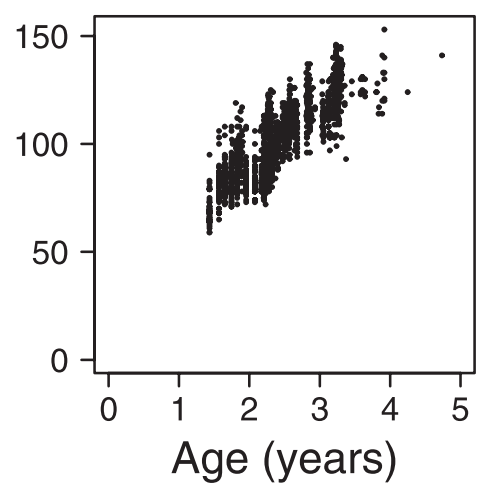

During the later part of the Girnock electrofishing campaign, brown trout (Salmo trutta) caught contemporaneously with the salmon parr were treated similarly. We use the resulting series of length-at-age observations for the 2002 trout hatch in the Girnock Burn (Fig. 2b) as one of our test data sets.

During 2003, staff from the Centre for Ecology and Hydrology carried out an analogous series of electrofishing surveys on the brown trout population of the River Frome - a lowland river in the south part of the county of Dorset, England $\left(50^{\circ} 40^{\prime} \mathrm{N}, 2^{\circ} 15^{\prime} \mathrm{W}\right)$. Although this series of observations did not recapture any single cohort throughout its lifetime, we have been able to compile a composite series of 
length-at-age observations (Fig. 2a) covering ages from approximately 6 months to 4 years.

\section{Length-at-age statistics}

To fit this data, we derive values of the mean and $\mathrm{CV}$ for length at a series of ages. To minimize uncertainty, we need to make each sample as large as possible, but the number of animals observed at any exact age value is small, which implies the need to pool the observations. After some experimentation, we found that a window of 50 days (i.e., observations at a nominal 500 days represent ages from 475 to 525 days) gave the best results. For each sample, we determined the mean and $\mathrm{CV}$ of length at (nominal) age and then estimated the uncertainty in each derived value by constructing 1000 bootstrap resamples in the manner described by Davison and Hinkley (1997) (the results are shown in Fig. 3).

\section{Data fitting}

For reasons of parsimony, we assume that the mortality suffered by the growing cohort is length-independent, so we can fit the variant of the model defined by eqs. 15 and 17. The analysis of this model formulation in the Introduction section showed that any specific trajectory produced with parameters $\{\mu, p, \tau\}$ from a given initial condition can also be produced by other values of this parameter triplet. To obtain a one-to-one relationship between trajectories and parameters, we must use additional evidence to select an appropriate value for one of the three parameters. In this case, we have no such additional evidence, so we arbitrarility set $\tau$ to a convenient value, namely $\tau=10$ days.

Comparing the data (Fig. 3) with the trajectories that this model predicts for a cohort growing under constant conditions (Fig. 1) shows that all three pairs of age-length and age-CV trajectories contain features that cannot be predicted by eqs. 15 and 17 with constant parameters. Specifically, each pair of trajectories exhibits repeated episodes in which the rate of increase of the mean and the rate of decrease of the CV both rise sharply, remain high for a short period, and then fall back. These episodes recur at intervals of almost exactly 365 days, so they appear to affect all year classes simultaneously in the Frome trout data and also affect the year classes we observe in successive years in the Girnock at the same time each year.

In the model defined by eqs. 15 and 17, increasing the growth probability, $p$, increases the rate of increase of the mean and the rate of decrease of the CV. A natural way to model the growth pattern shown in Fig. 3 is thus to set $p$ to a high value (say $p_{g}$ ) during some growing season and to a lower value ( $\operatorname{say} p_{w}$ ) during the rest of the year. We define age as elapsed time from 1 January in the year of hatch, so an individual is aged $a$ on a day of the year $\left(T_{y}\right)$ given by

$$
T_{y}(a)=a \bmod 365
$$

We shall regard the growing season as extending from a time of year $U$ days from 1 January to a time $D$ days from the same datum. For fitting purposes, we require a continuous relation among $U, D$, and $p$. To achieve this, we derive the value of $p$ for an update, which increments age from $a \rightarrow$ $a+\tau$ from a piece-wise continuous function, which changes linearly from $p_{w}$ to $p_{g}$ between $U$ and $U+\tau$ and returns linearly from $p_{g}$ to $p_{w}$ between $D$ and $D+\tau$, i.e.

$$
p= \begin{cases}p_{w} & \text { if } T_{y}(a)<U \text { or } T_{y}(a)>D+\tau \\ p_{g} & \text { if } T_{y}(a)>U+\tau \text { and } T_{y}(a)<D \\ p_{w}+\left(p_{g}-p_{w}\right)\left[T_{y}(a)-U\right] / \tau & \text { if } T_{y}(a) \geq U \text { and } T_{y}(a)<U+\tau \\ p_{g}-\left(p_{g}-p_{w}\right)\left[T_{y}(a)-D\right] / \tau & \text { if } T_{y}(a) \geq D \text { and } T_{y}(a)<D+\tau\end{cases}
$$

The full list of optimizable parameters for the extended model defined by eqs. 15, 17, and 22 is given in Table 1 .

To fit this model to one of the data sets shown in Fig. 3, we define a single observation objective as the ratio of the absolute difference between predicted and observed values to the range between the $95 \%$ confidence limits, a trajectory objective as the mean of the observation objectives for all the observed points on that trajectory, and the overall objective as the mean value of the two trajectory objectives.

The parameter set is optimized by minimizing the overall objective with predicted trajectories starting at the time of the first observation, with mean length set to $\hat{L}_{i}$ and length $\mathrm{CV}$ set to $c_{i}$. The optimization process uses the method of boostrap restarting advocated by Wood (2001). This begins with a basic optimization (in this case using the NelderMeade algorithm implemented in the R routine "optim") from an arbitrary starting point to achieve a starting parameter set and objective value. It proceeds through a series of cycles in which the same objective is minimized over a set of data generated from a bootstrap resample of the original observations; the resulting parameter set is used as the starting point for a new optimization on the target data. If this process produces a lower objective than the current best value, its parameters become the current best estimate of the fit parameters and the accompanying objective becomes the new target value. We repeated this cycle 10 times to obtain optimal parameters from a given start point and repeated the whole process 100 times from randomly chosen start points, with the overall lowest objective being used to define the best-fit parameters given in Table 1.

The quality of fit implied by the best-fit parameters for the three data sets is illustrated (Fig. 4). We see that the 
Fig. 3. Length-at-age statistics for wild salmonids: ( $a$ and $b$ ) brown trout (Salmo trutta) observed during 2002-2003 in the River Frome, $(c$ and $d$ ) trout from the 2002 hatch in the River Girnock, ( $e$ and $f$ ) Atlantic salmon (Salmo salar) parr from the 1997 hatch in the River Girnock. Panels $a, c$, and $e$ show mean length-at-age (points determined from data pooled in 50-day windows) together with 95\% confidence limits (dotted lines determined from 1000 bootstrap resamples per window). Panels $b, d$, and $f$ show the coefficient of variation $(\mathrm{CV})$ for length-at-age (points) and 95\% confidence limits (dotted lines) determined for the same windows.

Mean

(a)

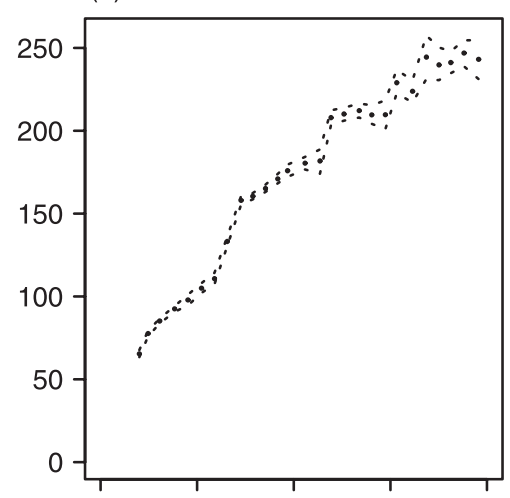

(c)

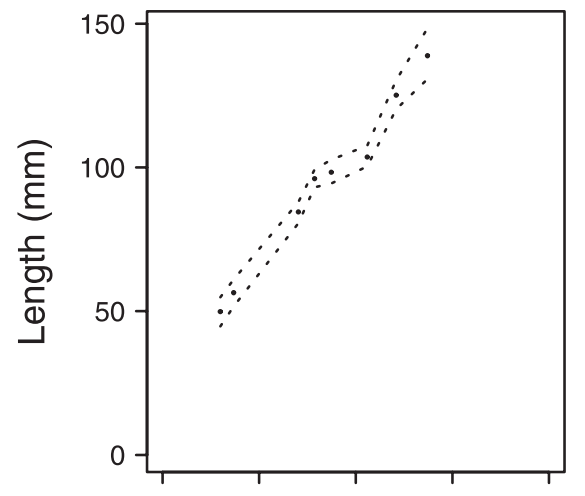

(e)

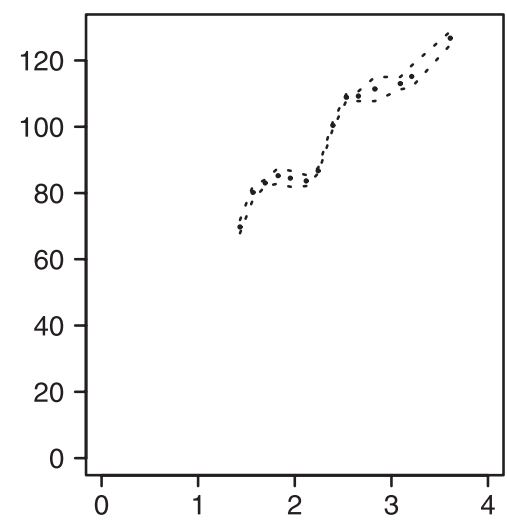

(b)

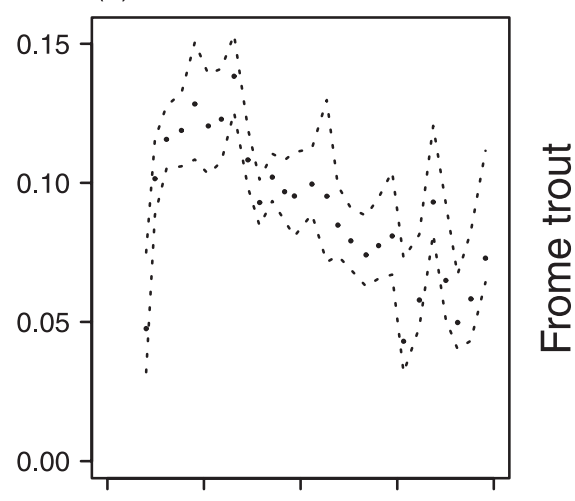

(d)

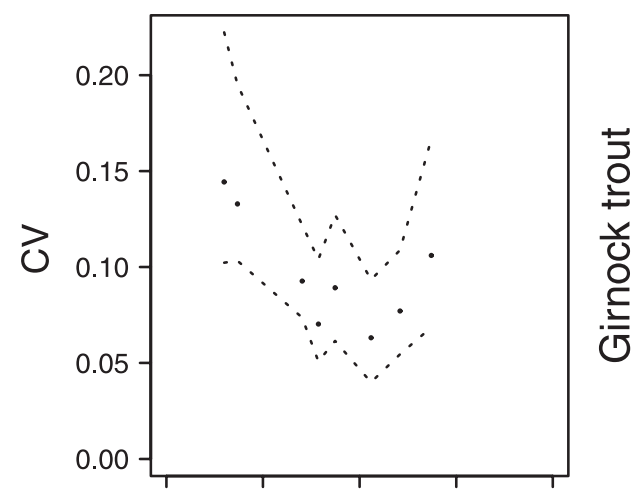

(f)

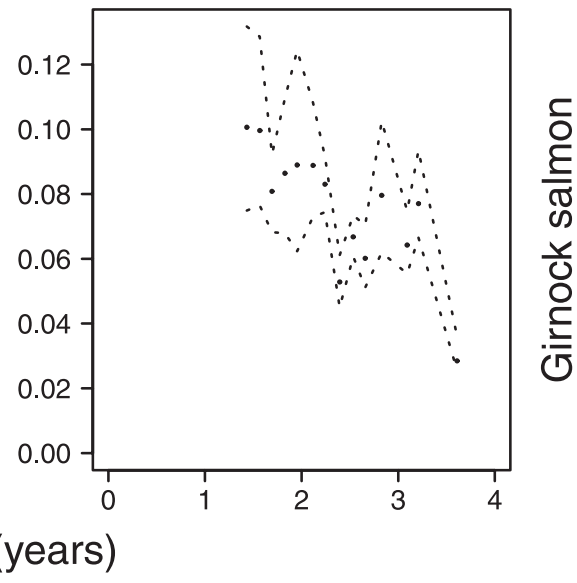

extended model captures the key features of all three data sets - namely the rapid increase in mean length during the yearly growing season accompanied by a substantial drop in variability, the downward trend in variability with increasing age, and the saturating (von Bertalanffy) form of the mean length curves leading to year-on-year decreases with age in the size of the yearly growth increment.

To obtain confidence limits for the best-fit parameters, we again used the method of bootstrap resampling (Davison and
Hinkley 1997). We first created a group of 500 pairs of trajectories of mean length-at-age and length-at-age $\mathrm{CV}$ by resampling the original set of observations and then recalculating the length-age statistics. We refitted each of these new target data sets using an objective function identical to that used in the determination of best-fit parameters, except that to minimize computational effort, the single observation objective was defined as the ratio of the absolute difference between the predicted and observed values for the new data 
Table 1. Best-fit values for salmonid parameters fitted with size-independent mortality and the growth correlation time $(\tau)$ set to 10 days.

\begin{tabular}{|c|c|c|c|c|}
\hline \multirow[b]{2}{*}{ Parameter } & \multirow[b]{2}{*}{ Symbol (units) } & \multirow{2}{*}{$\frac{\text { Frome }}{\text { Trout }}$} & \multicolumn{2}{|c|}{ Girnock } \\
\hline & & & Trout & Salmon \\
\hline Initial mean length & $\hat{L}_{i}(\mathrm{~mm})$ & 66.7 & 50.7 & 69.7 \\
\hline Initial length $\mathrm{CV}$ & $c_{i}(\%)$ & 4.47 & 13.1 & 8.63 \\
\hline Asymptotic length & $L_{\infty}(\mathrm{mm})$ & 306 & 309 & 148 \\
\hline Maintenance rate & $\mu\left(\% \cdot\right.$ day $\left.^{-1}\right)$ & 0.456 & 0.122 & 0.746 \\
\hline Growth probability (summer) & $p_{g}$ & 0.697 & 0.872 & 0.604 \\
\hline Growth probability (winter) & $p_{w}$ & 0.149 & 0.152 & 0.023 \\
\hline Growing season start & $U$ (day of year) & 71.9 & 39.6 & 72.3 \\
\hline Growing season end & $D$ (day of year) & 147 & 186 & 188 \\
\hline
\end{tabular}

Note: Trout refers to brown trout (Salmo trutta); salmon refers to Atlantic salmon (Salmo salar).

set to the confidence limit range for the equivalent point in the original data set. Each refit process was started from a single, randomly chosen point and restricted to 10 bootstrap restarts per data set. From the resulting 500 refits, we selected those that achieved an overall objective within $10 \%$ of the best-fit objective for the original data set and used this sample to determine $95 \%$ confidence limits (Table 2). In addition, we checked the fitted parameter sets for pair-wise correlations. Most pairs were not significantly correlated, and even in those few cases where statistically significant correlations were found, the proportion of the observed variability explained was small.

Comparing the best-fit values and confidence limits (Tables 1 and 2) shows the results of this fitting exercise to be consonant with our biological and statistical expectations. The Frome data set is longer, less noisy, and has much more in the way of distinctive pattern than those from the Girnock, so we are unsurprised to notice that the fitting process has defined the parameters much better in this case. The Girnock trout data set is the shortest and noisiest of the three, and we are thus equally unsurprised to observe that the fitted parameters are the most poorly defined.

Nonetheless, we can see that $L_{\infty}$, which we expect to depend principally on the basic species physiology, is indistinguisable between the two trout data sets and significantly lower in the case of the salmon parr.

Since the Frome is a lowland river some $400 \mathrm{~km}$ south of the Girnock, we are similarly encouraged to observe considerable differences in trout maintenance rates $(\mu)$, with lower values in the Girnock than in the Frome. In the Girnock, the maintenance rate for salmon parr is substantially greater than that for trout.

Surprisingly, the start of the growing season turns out to be essentially site- and species-independent, while its end clearly depends on site (but not species), being later in the high northerly Girnock and earlier in the low southerly Frome.

\section{Atlantic cod on the European continental shelf}

\section{The data}

The IBTS are multinational progammes to determine the spatial distribution of a number of demersal species, including Atlantic cod (Gadus morhua), over shelf seas, such as the North Sea, between the UK and the European mainland. The main activity of these surveys involves standard trawl tows using a carefully defined protocol (IBTS Working Group 2004), with the catch being length-classified and then counted. To permit the resolution of this length-classified data into year classes, otoliths are collected from a (nominally fixed) number of representatives of each length class and analysed to determine the age of the animal (modulo 1 year) and hence its year class.

The IBTS otolith data provides us with a set of individuals for whom we know both length and age (as defined in the previous section, namely time from 1 January in the year of hatch). However, the protocol used to select the subsample from which otoliths are collected means that its length-at-age distribution is very unlikely to be the same as that of the population at large.

To make proper use of the IBTS age data, we must employ it in the way the survey designers intended. We first pool the otolith data for all years over some region and intrayear period, so as to obtain a sensible number of otoliths for each length class. The pooled otolith data is then used to determine the proportional age-class composition of each length class at that time of year. This in turn allows us to derive the absolute age distribution for fish of each length class in every haul within that spatial area at the time of year of interest. Extracting the abundance of any given age class from all length classes present in a haul then gives us the length distribution for that age in that haul. Since this distribution is based on otolith data pooled across times of year and spatial locations, it is consistent to finally pool the derived length-at-age distributions across the same spatial area and equivalent age widows.

We show (Fig. 5) length-at-age statistics derived from IBTS data for the period 1991-1999 pooled temporally by quarter and spatially for the German Bight $\left(53.5^{\circ} \mathrm{N}, 3^{\circ} \mathrm{E} \times\right.$ $\left.55.5^{\circ} \mathrm{N}, 8^{\circ} \mathrm{E}\right)$, the Jutland Bank $\left(55.5^{\circ} \mathrm{N}, 3^{\circ} \mathrm{E} \times 57.5^{\circ} \mathrm{N}\right.$, $\left.8^{\circ} \mathrm{E}\right)$, and Viking $\left(60^{\circ} \mathrm{N}, 1^{\circ} \mathrm{E} \times 62^{\circ} \mathrm{N}, 7^{\circ} \mathrm{E}\right)$.

\section{Data fitting}

In this section, we again make the parsimonious assumption that mortality is size-independent. Although the yearly environmental cycle experienced by demersal fish such as cod is likely to be less dramatic than that to which salmon parr are exposed, one would nonetheless expect season variations in their rate of growth. However, the temporal resolu- 
Fig. 4. Fitted length-at-age statistics for wild salmonids. Panels $a$, $c$, and $d$ show fitted values (solid lines) and 95\% confidence limits for observed values (dotted lines) of mean length-at-age. Panles $b, d$, and $f$ show fitted values (solid lines) and $95 \%$ confidence limits for observed values (dotted lines) of coefficient of variation (CV) for length-at-age. Panels $a$ and $b$ refer to brown trout (Salmo trutta) observed in the River Frome in 2002-2003, $c$ and $d$ refer to the 2002 trout hatch in the River Girnock, and $e$ and $f$ refer to the 1997 Atlantic salmon (Salmo salar) parr hatch in the River Girnock.

\section{Mean}

(a)

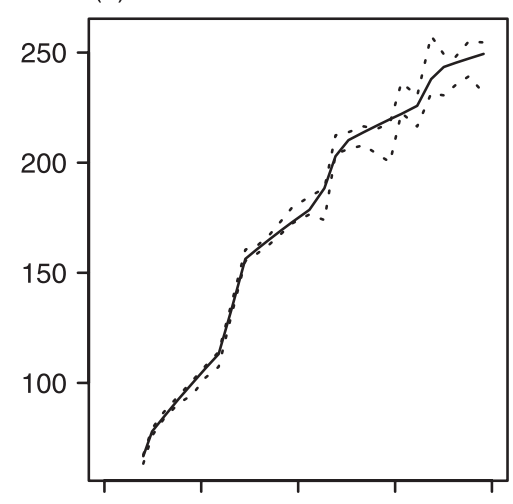

(c)

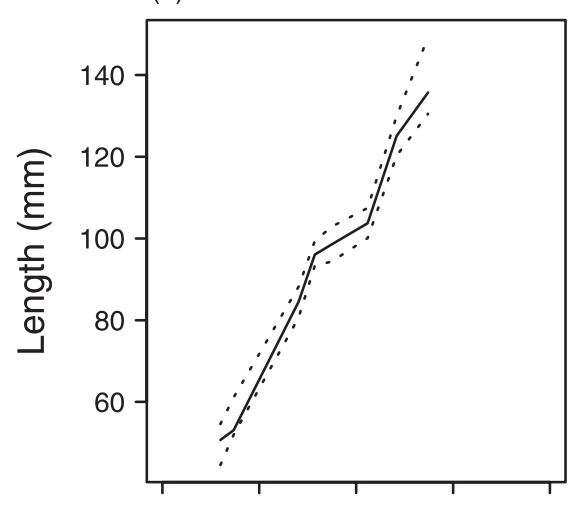

(e)

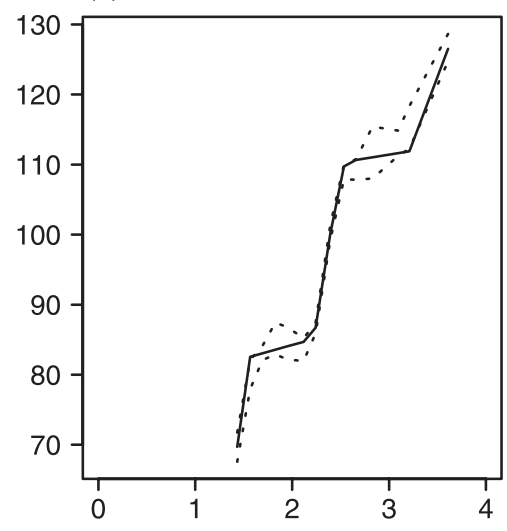

(b)

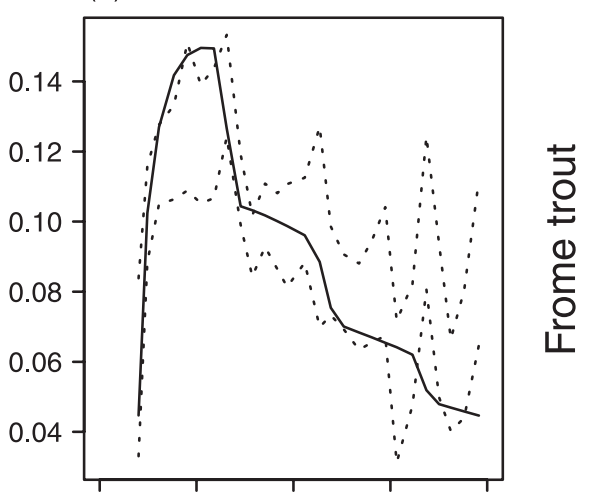

(d)

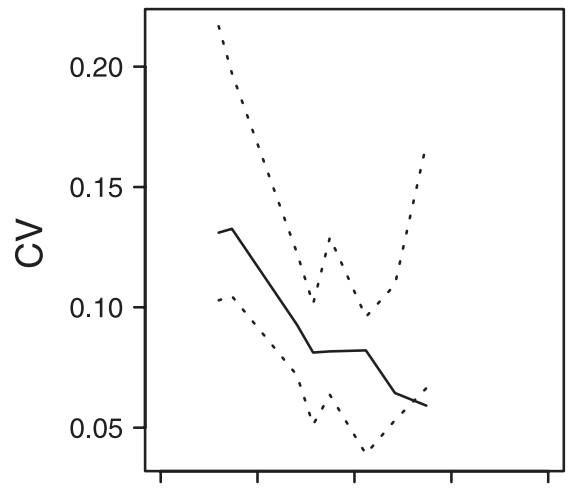

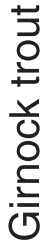

(f)

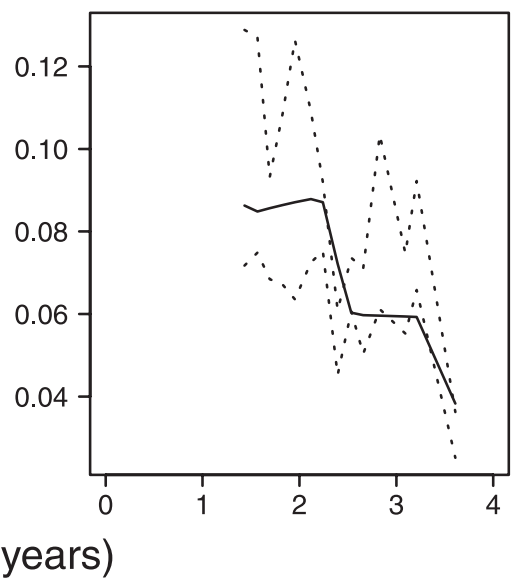

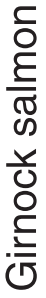

tion of our test data set is insufficient to allow unequivocal identification of a growing season, so we fit the basic model defined by eqs. 15 and 17 , with $\tau$ arbitarily set to a convenient value ( $\tau=10$ days).

Using the same fitting protocol as before, we obtain the best-fit parameters shown in Table 3 . We show the quality of fit implied by these parameters (Fig. 6) and see that with the exception of a possible misfit to the average length-at-age curves for individuals over 5 years old, the fitted model successfully captures the broad features of all three target data sets - namely the saturating character of the mean lengthat-age and the concave downward trend of the CV.

Confidence limits for the parameters in Table 3, obtained using exactly the same protocol before, are shown in Ta- 
Table 2. 95\% confidence limits for salmonid parameters fitted with size-independent mortality and $\tau=10$ days, obtained from 500 bootstrap resamples of the original observations.

\begin{tabular}{|c|c|c|c|c|}
\hline \multirow[b]{2}{*}{ Parameter } & \multirow[b]{2}{*}{ Symbol (units) } & \multirow{2}{*}{$\frac{\text { Frome }}{\text { Trout }}$} & \multicolumn{2}{|l|}{ Girnock } \\
\hline & & & Trout & Salmon \\
\hline Initial mean length & $\hat{L}_{i}(\mathrm{~mm})$ & $64.1-68.2$ & $47.7-54.1$ & $68.3-72.3$ \\
\hline Initial length $\mathrm{CV}$ & $c_{i}(\%)$ & $1.52-5.38$ & $8.49-15.1$ & $6.10-10.1$ \\
\hline Asymptotic length & $L_{\infty}(\mathrm{mm})$ & 299-322 & $186-345$ & $143-159$ \\
\hline Maintenance rate & $\mu\left(\% \cdot\right.$ day $\left.^{-1}\right)$ & $0.391-0.498$ & $0.110-0.451$ & $0.530-0.981$ \\
\hline Growth probability (summer) & $p_{g}$ & $0.619-0.800$ & $0.702-1.00$ & $0.561-0.864$ \\
\hline Growth probability (winter) & $p_{w}$ & $0.126-0.167$ & $0.014-0.218$ & $0.017-0.066$ \\
\hline Growing season start & $U$ (day of year) & $58.0-83.2$ & $30.1-103$ & $71.1-99.6$ \\
\hline Growing season end & $D$ (day of year) & $146-156$ & $153-234$ & $167-193$ \\
\hline
\end{tabular}

Note: Trout refers to brown trout (Salmo trutta); salmon refers to Atlantic salmon (Salmo salar).

ble 4. In contrast with our earlier example, a number of pairwise correlations can be discerned in the results of this refitting exercise (the most important of these are illustrated in Fig. 7).

Since the data sets in this group are clearly noisier than the salmonid data and contain much less in the way of finescale detail that the model might fit, we might expect to to see that the parameters of these fits are less well defined than those for the salmonids. However, with the exception of the asymptotic length $\left(L_{\infty}\right)$, all the parameters are little more uncertain than their salmonid equivalents.

Within the obvious limitations of uncertainties and correlations, we can see some quite well-defined geographical patterns. The mean and variability of the length at first observation for Viking and the German Bight are almost indistinguishable, with the mean for fish first observed on Jutland Bank being clearly lower. This is consistent with the $\mu$ values whose confidence intervals largely overlap for Viking and the German Bight, but are almost certainly lower for fish caught on Jutland Bank. This pattern is even clearer when we take account of the correlations in Figs. $7 g-7 i$, where we see that the values on the $\mu-p$ plane are clearly overlapping for Viking and the German Bight, but fall into a distinct region much nearer the origin for the Jutland Bank. This tells us that the product $\mu p$ and hence the expected relaxation rate (cf. eq. 16) is substantially lower on the Jutland Bank than in the other two locations.

\section{Discussion}

\section{The modelling framework}

In this paper, we describe a discrete-time framework for modelling the size-distribution dynamics of a population of organisms exhibiting irreversible von Bertalanffy growth. This formalism is closely related to the continuous-time framework proposed by Gurney and Veitch (2007) but differs from it in two key regards: first, it is an exact representation of a growth process whose rate must be guaranteed non-negative; and second, it implies a highly efficient numerical scheme, thus making it more suited to data fitting.

The von Bertalanffy (1938) model is a deterministic growth model, widely used in both assessment and management of aquatic and terrestrial populations, which can be derived from a rigorous picture of the assimilation and allo- cation of energy. Our stochastic extension preserves this mechanistic perspective. It assumes that all individuals exhibit the same size-dependent functional response and show randomly varying growth rates in response to stochastic variations in food availability. The key to the simplicity of our approach is the modelling approximation that an individual's growth rate can be regarded as constant over a period $\tau$ (which we call the growth correlation time) and that the average food availability over this period will either be high enough to imply ingestion (and hence growth) at the physiological maximum rate or low enough to imply zero growth. We assume that these two eventualities occur with probability $p$ and $1-p$, respectively.

The basic formalism (eq. 10) is readily applicable to modelling the long-term dynamics of a size-structured population, but in this paper we concentrate on applications that describe the changes in size distribution that accompany the growth of a single cohort. The proximate aim of this work is a framework within which we can understand age-structured size-distribution observations.

Although the principal aim of the formalism is accurate representation of non-negative growth combined with computational efficiency, we have made some progress with analytic and semianalytic treatments. In the special case where the per-capita mortality rate does not depend specifically on size, we derive update rules for the first two moments of the length-at-age distribution, which provide an exceptionally efficient route to numerical evaluation of predicted trajectories. We note that the trajectories predicted by these rules depend only on the first two moments of the initial lengthat-age distribution and are entirely independent of its specific form. We also note that in this approximation, the predictions are entirely independent of the magnitude of the mortality and of its dependence on age or time.

In the same special case, we show that an attracting trajectory of length-at-age $\mathrm{CV}$, of the type described by Gurney and Veitch (2007), exists in the model described here, and we obtain a closed form approximation to it. This approximation is too crude to be useful in error minimization, but it gives a very clear idea of how the model's dynamics depend on its parameters - a very important consideration in the early stages of an investigation, where the question at issue is often the ability of the model to produce trajectories that exhibit the same qualitative properties as those shown by the 
Fig. 5. Observed length-at-age statistics for Atlantic cod (Gadus morhua) in the North Sea during the 1990s: ( $a$ and $b$ ) German Bight, $(c$ and $d$ ) Jutland Bank, ( $e$ and $f$ ) Viking. Panels $a, c$, and $e$ show mean length-at-age (points determined from data pooled in quarteryear windows) together with 95\% confidence limits (dotted lines determined from 1000 bootstrap resamples). Panels $b, d$, and $f$ show the coefficient of variation $(\mathrm{CV})$ for length-at-age (points) and 95\% confidence limits (dotted lines).

Mean

(a)

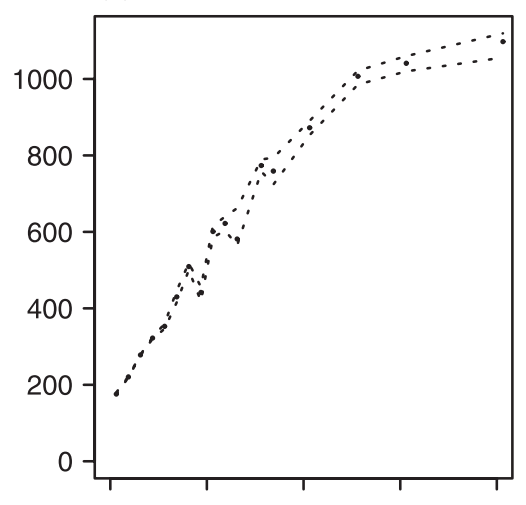

(c)

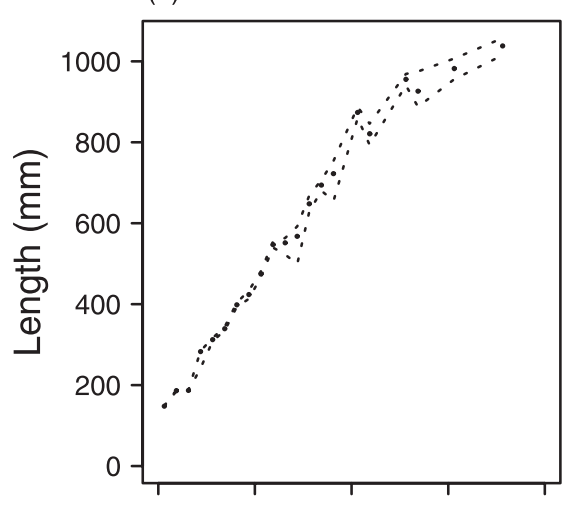

(e)

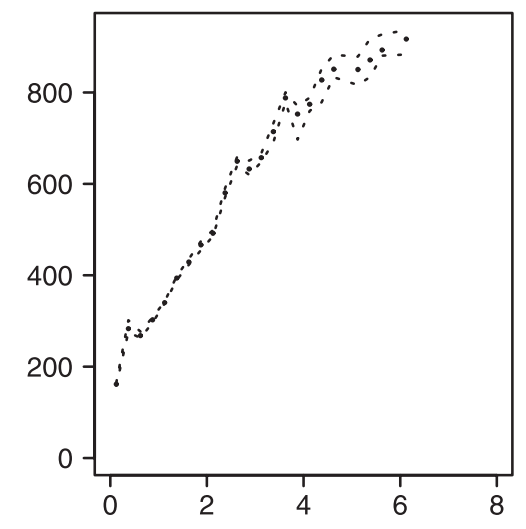

(b)

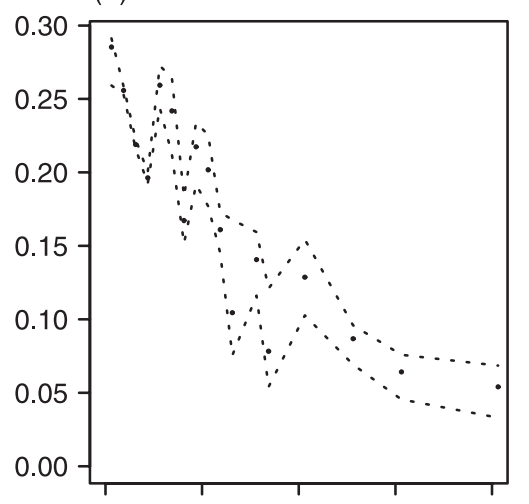

(d)

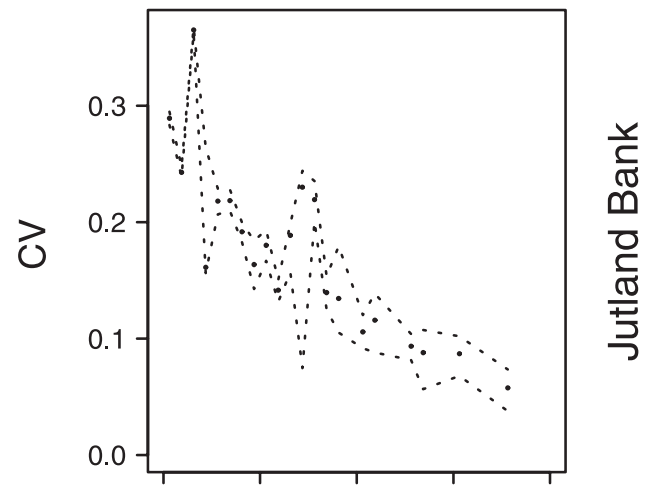

(f)

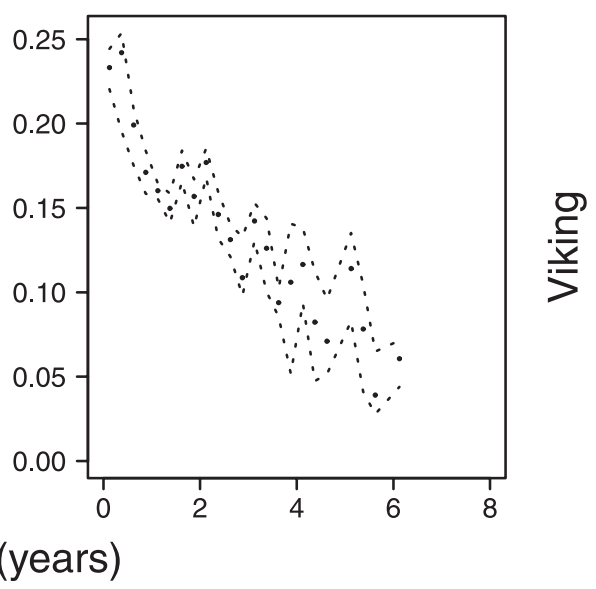

data. We find that where the product of the growth correlation time $(\tau)$ and the length-specific maintenance rate $(\mu)$ is small, the quasi-stationary length $\mathrm{CV}$ is proportional to the product of $\sqrt{(1-p) \mu \tau}$ and the difference between the current and asymptotic mean lengths. This implies that the lengthat-age $\mathrm{CV}$ of a cohort goes to 0 as the mean length of its surviving members approaches $L_{\infty}$, with the rate of approach being dependent on $(1-p) \mu \tau$. This in turn suggests that a fit to both the mean and CV of length-at-age will be more sen- sitive to $L_{\infty}$ than a fit to the mean alone and will thus give a reasonable estimate of the parameters even where (as for salmon parr) no major proportion of the cohort reaches a length close to the asymptotic value.

We illustrate the utility of this framework by using it to fit two contrasting data sets describing the size-at-age of commercially interesting fish populations. In one of these cases, we have to make a minor structural extension to the submodel of growth rate variability before obtaining a satisfac- 
Table 3. Best-fit values for Atlantic cod (Gadus morhua) parameters fit with sizeindependent mortality and growth correlation time $(\tau)$ set to 10 days.

\begin{tabular}{lllll}
\hline Parameter & Symbol (units) & German Bight & Jutland Bank & Viking \\
\hline Initial mean length & $\hat{L}_{i}(\mathrm{~mm})$ & 163 & 142 & 162 \\
Initial length CV & $c_{i}(\%)$ & 28.5 & 28.9 & 23.3 \\
Asymptotic length & $L_{\infty}(\mathrm{mm})$ & 1750 & 2740 & 1660 \\
Maintenance rate & $\mu\left(\% \cdot\right.$ day $\left.^{-1}\right)$ & 0.176 & 0.0965 & 0.142 \\
Growth probability & $p$ & 0.23 & 0.195 & 0.254 \\
\hline
\end{tabular}

tory fit. The ease with which this can be achieved illustrates the extensibility of the approach and hence the width of its potential applicability.

It is important to stress that these two exemplary applications are intended as demonstrations, rather than as exhaustive investigations. We thus fit a specific model and demonstrate that it can produce a respectable fit to the data rather than make a comparative investigation of a family of candidate models.

In a similar vein, we neglect size-specific mortality, despite frequent demonstrations in the literature (see Gudmundsson 2005 and references therein) of the importance of size- (or age-) dependent mortality in determining observed population size distributions. We investigate the likely sensitivity of the fitting process to this omission below.

\section{Exemplary application - salmonids in two UK rivers}

Our first example concerned relatively well-defined cohorts of juvenile salmonids growing in one Scottish and one English river. Because the riverine environment shows a very marked yearly cycle and our data has sufficient temporal resolution to resolve the resulting growth patterns, we needed to recognise that the growth probability $p$ varies within a year. With the aid of a very simple picture, which assumed that $p$ takes the same two distinct values within fixed periods (broadly growing and nongrowing seasons) of all years, we obtained very satisfactory fits to subsets of the data covering two species (salmon and trout) and two locations (in upland Scotland and lowland England).

Although the mortality of salmon parr is generally only weakly dependent on size (or age), this species undergoes obligate emigration to sea before completing its life cycle. This process is highly size-dependent - being virtually unknown for parr $<70 \mathrm{~mm}$ long and almost certain for parr $>20 \mathrm{~mm}$ long. A realistic model of this process is well beyond the scope of this paper, but we can crudely simulate its effects on the size distribution of the river population by introducing an additional mortality rate $\left(\rho_{c}\right)$, which applies to individuals whose length exceeds some threshold $\left(L_{c}\right)$.

We show (Fig. 8) the results of three runs using parameters appropriate to the salmon population in the Girnock, with the threshold length $L_{c}=90 \mathrm{~mm}$, a background mortality rate set to $\rho_{b}=0.0022$ day $^{-1}$ (equivalent to a year-to-year survival of 0.5 ), and the additional mortality rate $\rho_{c}$ set to 0 , 0.008 , and $0.02 \mathrm{day}^{-1}$, respectively We see that although additional mortality of 0.008 day $^{-1}$ (equivalent to a yearly survival of 0.05 and thus to removing the emigrating individuals over a period of months) produces a major effect on the survival to age curve and will hence have an important effect on the stable size distribution of a population with continuous recruitment, it produces only very small effect on the mean length-at-age and CV-at-age curves. By contrast, an additional mortality of $\rho_{c}=0.02 \mathrm{day}^{-1}$ (equivalent to a year-to-year survival of 0.0007 and thus to removing all emigrating individuals over a period of days) alters the predicted length-at-age and CV-at-age curves by an amount that is comparable with the confidence limits shown in Fig. 3.

We thus argue that while quite small size dependence of mortality or emigration can produce very major changes in a population's stable size distribution, substantial change in the length-at-age statistics requires a much more severe size dependence of mortality. This is essentially because size dependence of mortality will only produce important changes in the length-at-age statistics of a cohort if the mortality rate varies considerably across the cohort's size distribution at any given age. This suggests that the conclusions drawn from the fitting exercise described here are likely to be relatively robust against a change to the simplifying assumption that mortality is size-independent.

\section{Exemplary application - Atlantic cod in European shelf waters}

Our second example involved more complex and difficult data, concerning Atlantic cod on the European shelf. The survey program that provided the data was primarily designed to measure spatial variations in size distribution and secondarily to define the age distribution (modulo 1 year) of individuals of a given size. The implication of this survey design is that the animals whose ages we know do not form a random sample from the population, so their size-at-age distribution is not representative of that of the population at large. By pooling data over wide spatial and temporal ranges and accepting low age resolution $(0.25$ years $)$, we obtained a defensible picture of mean and $\mathrm{CV}$ of length-at-age in three contrasting locations (the German Bight, the Jutland Bank, and sea-area Viking).

In view of the generally noisier character of this data, as well as its lower temporal resolution, we fit it using our basic model with constant coefficients. Although the resulting fits clearly capture almost all of the features of the data that such a model might be expected to reproduce, we note that several data show evidence of poor fidelity at the highest ages, accompanied by values for the asymptotic length, which, while not entirely implausible, are still somewhat high. The technical reason for this is that the model does not permit the further reduction of $L_{\infty}$, which would improve the fit to the mean at high ages without disproportionate reductions in $\mathrm{CV}$ in the same age range. One possible cause for strong saturation in mean without a commensurate reduction in $\mathrm{CV}$ would be that the population is not composed of a 
Fig. 6. Fitted length-at-age statistics for Atlantic cod (Gadus morhua) in the North Sea in the 1990s. Panels $a$, $c$, and $d$ show fitted values (solid lines) and 95\% confidence limits for observed values (dotted lines) of mean length-at-age. Panels $b, d$, and $f$ show fitted values (solid lines) and 95\% confidence limits for observed values (dotted lines) of coefficient of variation (CV) for length-at-age. Panels $a$ and $b$ refer to observations in the German Bight, $c$ and $d$ refer observations on the Jutland Bank, and $e$ and $f$ refer observations in Viking.

\section{Mean}

(a)

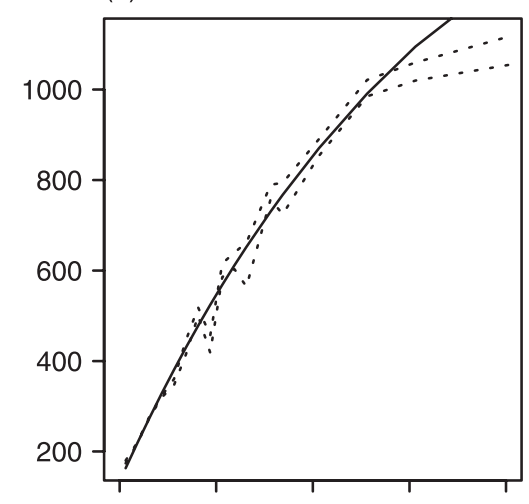

(c)

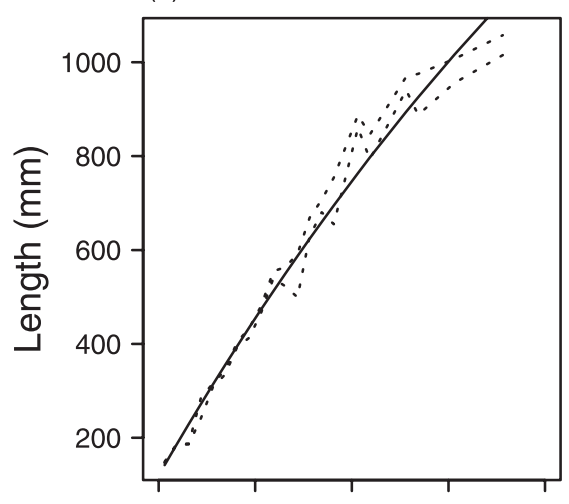

(e)

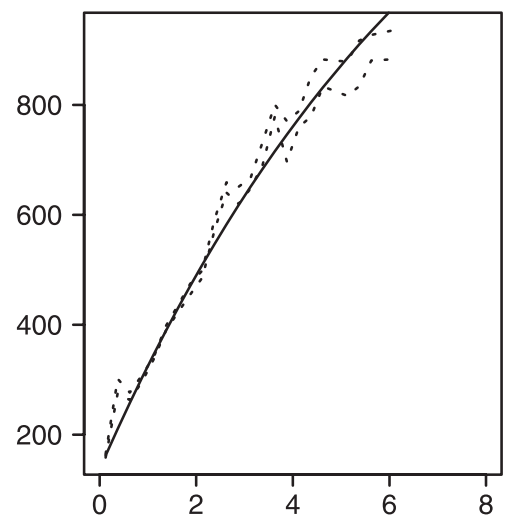

(b)

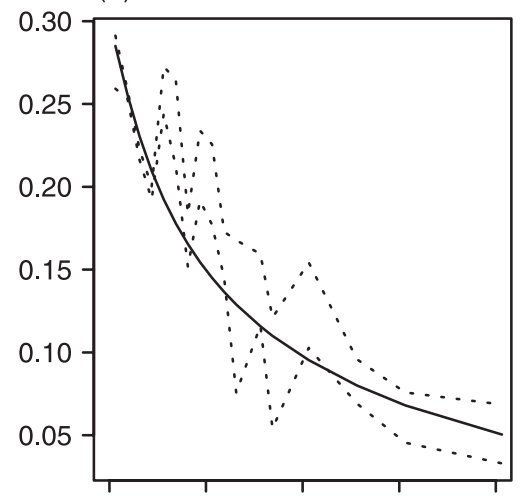

(d)

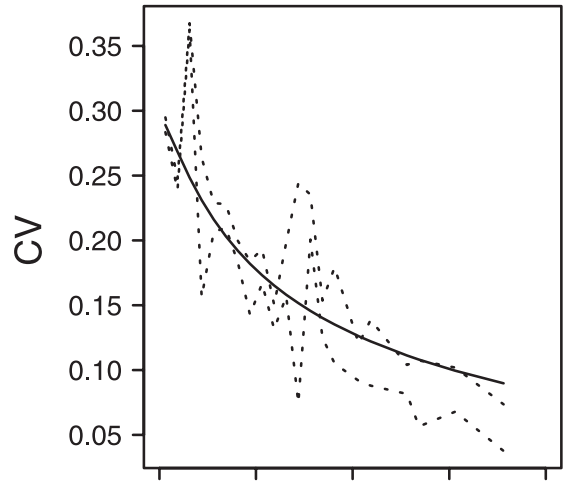

(f)

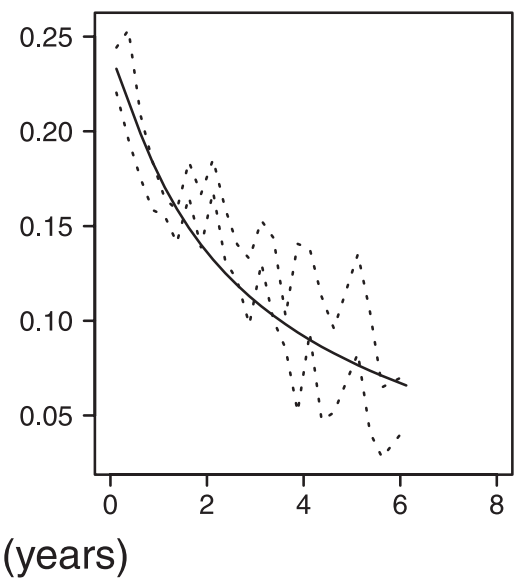

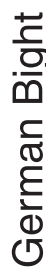

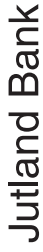

$\frac{\text { 곧 }}{>}$ single group of statistically similar individuals, but instead is composed of a collection of groups of individuals (families, sensu Gurney and Veitch 2007), each with distinct statistical properties. However, the data analysed here is too imprecise to distinguish this causal mechanism from other equally plausible candidates.

\section{Data fitting}

In both exemplary applications, we adopted the statistically controversial approach of fitting to derived quantities (mean and CV of length-at-age) rather than to raw data. Despite the undoubted statistical risks involved in such a course, we believe it to have worthwhile benefits. In our ex- 
Table 4. 95\% confidence limits for Atlantic cod (Gadus morhua) parameters fit with sizeindependent mortality and $\tau=10$ days, obtained by fitting 500 bootstrap resamples of the original data.

\begin{tabular}{lllll}
\hline Parameter & Symbol (units) & German Bight & Jutland Bank & Viking \\
\hline Initial mean length & $\hat{L}_{i}(\mathrm{~mm})$ & $160-166$ & $140-144$ & $158-164$ \\
Initial length CV & $c_{i}(\%)$ & $27.2-29.2$ & $28.4-29.5$ & $22.1-24.2$ \\
Asymptotic length & $L_{\infty}(\mathrm{mm})$ & $1540-1880$ & $2140-3320$ & $1500-1840$ \\
Maintenance rate & $\mu\left(\% \cdot \mathrm{day}^{-1}\right)$ & $0.150-0.218$ & $0.0760-0.140$ & $0.119-0.180$ \\
Growth probability & $p$ & $0.214-0.258$ & $0.174-0.204$ & $0.0 .223-0.276$ \\
\hline
\end{tabular}

Fig. 7. Parameter correlations for fits to the resampled Atlantic cod (Gadus morhua) data: panels $a$, $d$, and $g$ refer to the German Bight; panels $b, e$, and $h$ refer to the Jutland Bank; and panels $c, f$, and $i$ refer to Viking. Panels $a-c$ show $\mu$ vs. $L_{\infty} ; d-f$ show $p$ vs. $L_{\infty} ; g-i$ show $p$ vs. $\mu$.
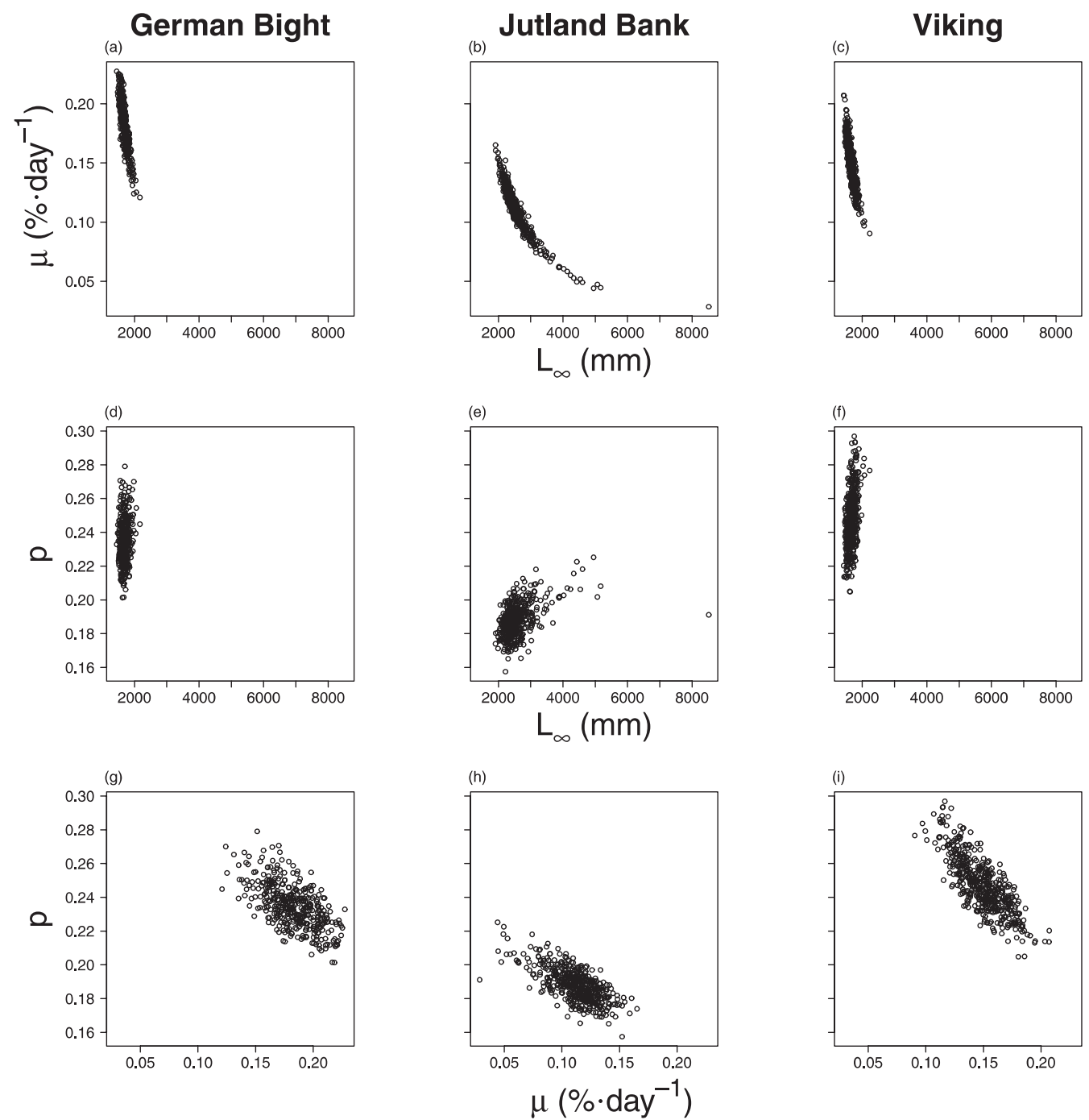

perience, the key stage in most model estimation processes is not the formal parameter estimation, but rather the elimination of a series of structurally inappropriate early candidate models. If this can be done without the labour of formal statsitical testing, considerable time and effort can be saved. Much of the power of the approach advocated in this paper comes from the fact that we have built up a major body of analysis linking the gross properties of the predicted mo- ment trajectories to model parameters and structure. Since these insights tell us about the qualitative properties of moment trajectories, it is natural to compare the best estimates we can make of such trajectories against those actually estimated from observations to discern whether the model is capable of producing trajectories of the required type. Once we have a candidate model that passes this test, we may usefully consider the deployment of a more comprehensive 
Fig. 8. Effects of size-dependent mortality are illustrated with three runs of a model describing the growth of Atlantic salmon (Salmo salar) parr in the River Girnock. The runs are defined by eqs. 10 and 22, with through-stage survival $S_{q}$ equivalent to a mortality rate $\rho_{b}=0.0022$ day $^{-1}$ for $L<L_{c}=90 \mathrm{~mm}$ and $\rho_{b}+\rho_{c}$ for $L \geq L_{c}$, where $\rho_{c}=0$ day $^{-1}$ (solid line), 0.008 day $^{-1}$ (dotted line), and 0.02 day $^{-1}$ (dashed line). (a) Mean length-at-age, $(b)$ length $\mathrm{CV}$-at-age, $(c)$ numbers-at-age, and $(d)$ mortality rate at size.

(a)

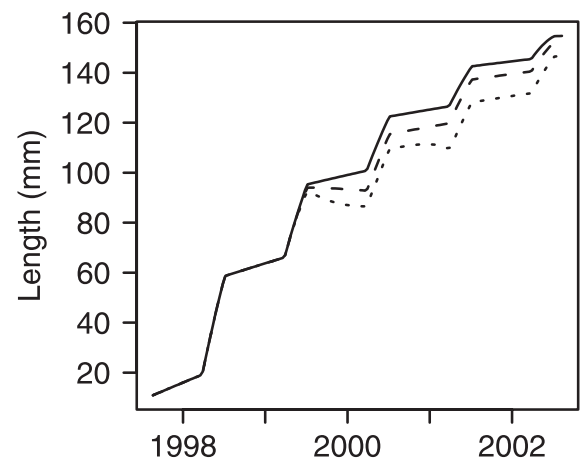

(c)

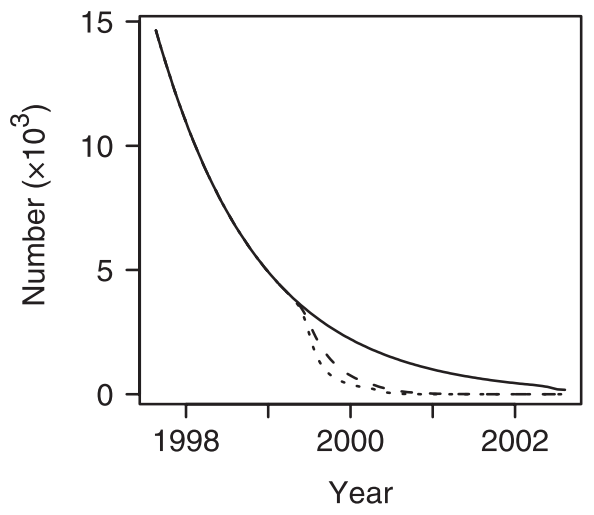

statistical armoury, including all the standard maximum likelihood and Markov chain Monte Carlo methods, in addition to the nonlinear minimization used in this paper.

On a less philosophical note, our analysis of the model variant with size-independent mortality has shown that although fitting to time series of simple summary statistics for cohort size-at-age (that is to values of mean length-at-age and length-at-age $\mathrm{CV}$ ) enables us to determine the maintenance rate $(\mu)$ and the realised proportion of potential growth (the growth probability $p$ ), we cannot determine the growth correlation time $(\tau)$ without additional evidence. In the absence of such evidence, a workable strategy is to choose a convenient but arbitrary value for $\tau$ that yields a fit to the available data with biologically plausible values for $\mu$ and $p$. Although we have only formally established this result for the case of size-independent mortality, our numerical experiments lead us to the belief that it applies equally to the more general case.

\section{Model extensions}

Although the exposition in this paper has concentrated on simulations of data characterizing a single cohort, the basic formalism (eq. 10) is equally suited to the description of a reproducing population. In this context, it provides a concep- (b)

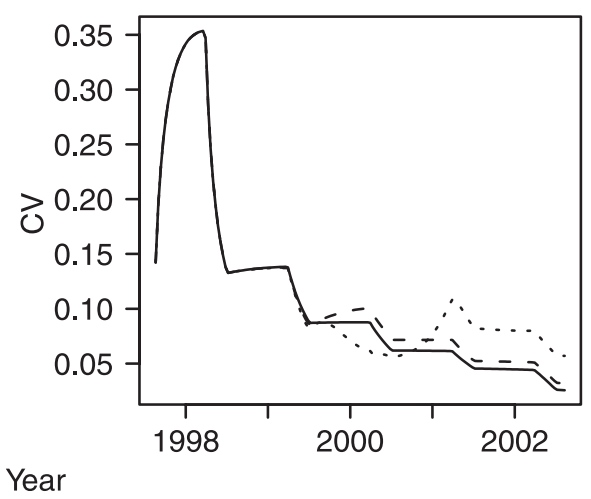

(d)

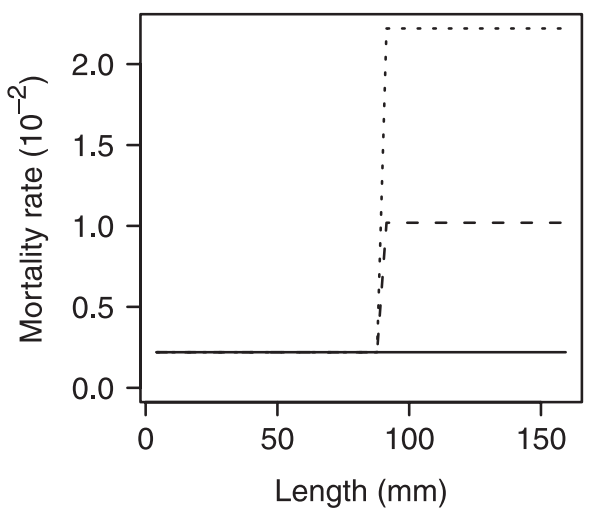

tually appealing and computationally convenient route to incorporating environmentally driven, individual-to-individual growth rate variability into population models without the need to resort to computationally expensive, individualbased methods.

Model extensions to improve biological realism are also possible. Perhaps the most important extension to the work described in our exemplary applications lies in the incorporation of strongly size-dependent mortality, such as that produced by size selectivity of the gears used in typical commercial fisheries. Our numerical experiments show that the effects of strongly size-dependent mortality can be surprisingly subtle, so prudent investigators will incorporate such effects only if they have very good observational evidence or if there are strongly marked patterns in the lengthat-age statistics, which require size-slective mortality for their explanation.

Although size-slective mortality can be included in the model without modification of eq. 10, other elements of biological realism require extensions to the formalism. For example, observed mean length-at-age curves for fish are often sigmoidal. Although we have treated the growth probability $p$ as being independent of length throughout this paper, there is no technical difficulty in the context of the full model 
(used in producing Fig. 8) in making $p$ an increasing function of length and hence producing a sigmoidal growth curve.

A further potentially important element of biological realism lies in incorporating the cost of reproduction into our discourse. Kooijman (1993) argues that expenditure on reproduction forms a constant tax on assimilation, which is paid throughout life - acting first to build gonads and later to operate them. In this picture, we simply incorporate the tax rate into eq. 4 , thus reducing $L_{\infty}$ by an appropriate factor, but leaving the basic shape of the growth curves unchanged.

If the reproduction tax rate varies through life - for example, being zero for immature animals and finite for matures - then matures and immatures would have different values of $L_{\infty}$ and hence different development indices. In such a case, the natural model extension would be to represent immatures by one development index array and matures by a second, with transitions between them determined by an appropriate maturation rule. Such an extension would produce characteristic "hockey-stick" deformations in the growth curves, and the occurance of such an otherwise inexplicable feature in data would be the cue for the investigation of such a model extension.

\section{Acknowledgements}

The authors acknowledge helpful conversations about the dynamics of size-at-age variability with Roger Nisbet and Douglas Speirs. We also thank two anonymous referees for comments on an earlier draft of this manuscript, which prompted useful improvements in statistical methodology, with important interpretational consequences.

\section{References}

Bacon, P.J., Gurney, W.S.C., Jones, W., McLaren, I.S., and Youngson, A. 2005. Seasonal growth patterns of wild juvenile fish: partitioning variation among explanatory variables based on individual growth trajectories of Atlantic salmon (Salmo salar) parr. J. Anim. Ecol. 74: 1-11.

Bardos, D.C. 2005. Probabilistic Gompertz model of irreversible growth. Bull. Math. Biol. 67: 529-545.

Buck, R.J.G., and Hay, D.W. 1984. The relationship between spawning stock and progeny of Atlantic salmon (Salmo salar) in a Scottish stream. J. Fish Biol. 23: 1-11.

Brunkow, P.E., and Collins, J.P. 1998. Group size structure affect patterns of aggression in larval salamanders. Behav. Ecol. 9: $508-514$.

Davison, A.C., and Hinkley, D.V. 1997. The basic bootstraps. In Bootstrap methods and their application. Edited by A.C. Davison and D.V. Hinkley. Cambridge University Press, Cambridge. pp. 27-30.

DeAngelis, D.L., and Huston, M.A. 1987. Effects of growth rates in models of size distribution formation in plants and animals. Ecol. Model. 36: 119-137.
DeAngelis, D.L., Cox, D.C., and Coutant, C.C. 1979. Cannibalism and size dispersal in young of the year large-mouth bass: experiments and model. Ecol. Model. 24: 21-41.

de Roos, A.M., Diekmann, O., and Metz, J.A.J. 1992. Studying the dynamics of structured population models: a versatile technique and its application to Daphnia population dynamics. Am. Nat. 139: $123-147$.

Fujiwara, M., Kendall, B.E., and Nisbet, R.M. 2004. Growth autocorrelation and animal size variation. Ecol. Lett. 7: 106-113.

Fujiwara, M., Kendall, B.E., Nisbet, R.M., and Bennett, W.A. 2005. Analysis of size trajectory data using an energetic-based growth model. Ecology, 86: 1441-1451.

Gompertz, B. 1825. On the nature of the function expressive of the law of human mortality, and on a new mode of determining the value of life contingencies. Philos. Trans. R. Soc. Lond. 115: 513-585.

Gudmundsson, G. 2005. Stochastic growth. Can. J. Fish. Aquat. Sci. 62: 1746-1755.

Gurney, W.S.C., and Nisbet, R.M. 1998. Ecological dynamics. Oxford University Press, New York.

Gurney, W.S.C., and Veitch, A.R. 2007. The dynamics of size-at-age variability. Bull. Math. Biol. 69(3): 861-885. doi:10.1007/s11538006-9167-8.

IBTS Working Group. 2004. Manual for the International Bottom Trawl Surveys. Revision VII [online]. International Council for the Exploration of the Sea, Copenhagen. Available from http:// www.ices.dk/datacentre/datras/NsIBTSmanualRevVIIdraft.pdf.

Jones, W., Gurney, W.S.C., Speirs, D.C., Bacon, P.J., and Youngson, A.Z. 2002. Seasonal patterns of growth, expenditure and assimilation in juvenile Atlantic salmon. J. Anim. Ecol. 71: 916-924.

Kooijman, S.A.L.M. 1993. Dynamic energy budgets in biological systems. Cambridge University Press, Cambridge.

Marteinsdottir, G., and Begg, G. 2002. Essential relationships incorporating influences of age, size and condition on variables required for estimation of reproductive potential in Atlantic cod Gadus morhua. Mar. Ecol. Progr. Ser. 235: 235-256.

Pfister, C.A., and Peacor, S.D. 2003. Variable performance of individuals: the role of population density and endogenously formed landscape heterogeneity. J. Anim. Ecol. 72: 725-735.

Pfister, C.A., and Stevens, F.R. 2002. The genesis of size variability in plants and animals. Ecology, 83: 59-72.

Ricker, W.E. 1958. Handbook of computations for biological statistics of fish populations. Fish. Res. Board Can. Bull. 119.

Rose, G. 2004. Reconciling overfishing and climate changes with stock dynamics of Atlantic cod (Gadus morhua) over 500 years. Can. J. Fish. Aquat. Sci. 61: 1553-1557.

Sebens, K.P. 1987. The ecology of indeterminate growth. Annu. Rev. Ecol. Syst. 18: 371-407.

von Bertalanffy, L. 1938. A quantitative theory of organic growth (inquiries on growth laws). II. Hum. Biol. 10: 181-213.

Wood, S.N. 2001. Minimising model fitting objectives which contain spurious local minima by bootstrap restarting. Biometrica, 57(1): 240-244.

Wootton, J.T. 1992. Indirect effects, prey susceptibility and habitat selection: impacts of birds on limpets and algae. Ecology, 73: 981-991. 